\title{
Probing the step structure of buried metal/semiconductor interfaces using quantized electron states: The case of $\mathrm{Pb}$ on $\mathrm{Si}(111) 6 \times 6-\mathrm{Au}$
}

\author{
Hongbin Yu and C.-S. Jiang \\ Department of Physics, University of Texas, Austin, Texas 78712 \\ Ph. Ebert \\ Institut für Festkörperforschung, Forschungszentrum Jülich GmbH, 52425 Jülich, Germany \\ C.-K. Shih ${ }^{\text {a) }}$ \\ Department of Physics, University of Texas, Austin, Texas 78712
}

(Received 25 February 2002; accepted for publication 17 July 2002)

\begin{abstract}
The three-dimensional step structure at the buried $\mathrm{Pb}$ on $\mathrm{Si}(111) 6 \times 6$-Au interface is determined by utilizing the presence of quantum well states. We demonstrate that the spatial step positions as well as the step heights can be extracted nondestructively and with atomic layer precision by scanning tunneling microscopy and spectroscopy. (C) 2002 American Institute of Physics.
\end{abstract}

[DOI: $10.1063 / 1.1506404]$

Thin metal films on semiconductor substrates are crucial components of many semiconductor devices. The physical properties of these thin films are to a large degree governed by the film morphology, which in turn is directly affected by the roughness of the buried metal-semiconductor interface. Despite large efforts in determining the interfaces' step structure with a variety of nondestructive tools, the real space three-dimensional step-structure determination of interfaces still remains a formidable task.

Recently, there has been much progress in synthesizing ultrathin metal films on semiconductor substrates. In particular, it has been shown that atomically flat thin metallic films with nanometer scale thickness can be successfully grown. ${ }^{1-5}$ In such an ultrathin metal film, electrons are subjected to strong confinement along the vertical direction, resulting in quantum well states (QWS). ${ }^{2}$ Indeed, such QWS have been observed using photoemission ${ }^{2,6,7}$ and scanning tunneling spectroscopy. ${ }^{8-11}$ Since the energy levels of QWS depend sensitively upon the film thickness, it is therefore possible, from the measurement of local QWS, to determine the local thickness of the thin film from which the underlying step structure can be deduced. ${ }^{12}$ Indeed, by studying individual $\mathrm{Pb}$ islands on $\mathrm{Si}(111)$ substrates, Altfelder et al., found that the variation of QWS correlates with the substrate step structure visible at the edges of the metal island. ${ }^{9}$ This correlation is, however, based on the ability to measure directly in scanning tunneling microscopy (STM) images the height of individual islands on the substrate (with wetting layer). Unfortunately such a simple correlation is no longer possible for a fully covering two-dimensional metal film, which is the ultimate goal for applications in semiconductor devices. In this letter we demonstrate the nondestructive methodology on how to determine the step structure at buried metal-semiconductor interfaces for fully covering twodimensional thin metal films using STM.

As a model system we grew $\mathrm{Pb}$ films on $\mathrm{Si}(111)$ $6 \times 6$ - Au surfaces at room temperature. On such a surface,

${ }^{a)}$ Electronic mail: shih@physics.utexas.edu
$\mathrm{Au}$ acts like an "interfactant" 13 and leads to atomically flat two-dimensional thin $\mathrm{Pb}$ films (such as the one shown in Fig. 1) and not to isolated islands as found for direct $\mathrm{Pb}$ growth on clean $\operatorname{Si}(111) 7 \times 7$ surfaces (see Ref. 9). Note that the surface steps visible in the $\mathrm{Pb}$ film in Fig. 1 are not connected in any way to the substrate steps. Thus, one cannot extract the step structure at the buried interface directly from the constant-current STM image. In order to extract the interface step structure we follow a different approach.

We measured on individual terraces of such surfaces constant current STM images simultaneously with $I-V$ spectra at every pixel point. Figure 2(a) shows such a constant current STM image of an $80 \times 80 \mathrm{~nm}^{2}$ area. The contrast visible arises from a superstructure (no atomic resolution). In addition two slightly darker lines are visible with a very subtle contrast (see arrows). They roughly divide the image

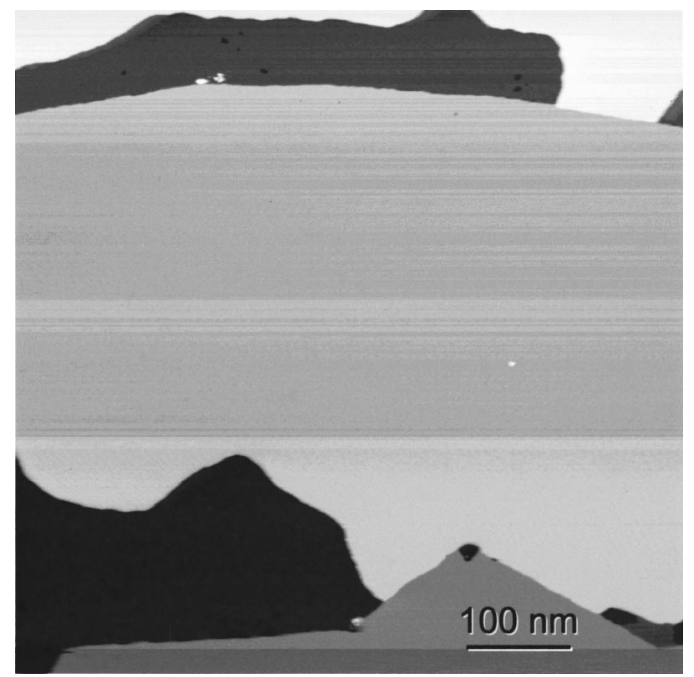

FIG. 1. Gray-scale constant-current scanning tunneling microscopy images of 3 monolayers $\mathrm{Pb}$ grown at room temperature on a $\mathrm{Si}(111) 6 \times 6-\mathrm{Au}$ surface. The surface exhibits large flat terraces of the $\mathrm{Pb}$ film, although steps on the $\mathrm{Si}$ substrates are buried below. Note that the $\mathrm{Pb}$ steps have no correlation with the steps at the buried interface. The linear gray scale from black to white covers a height change of $1.43 \mathrm{~nm}$. 

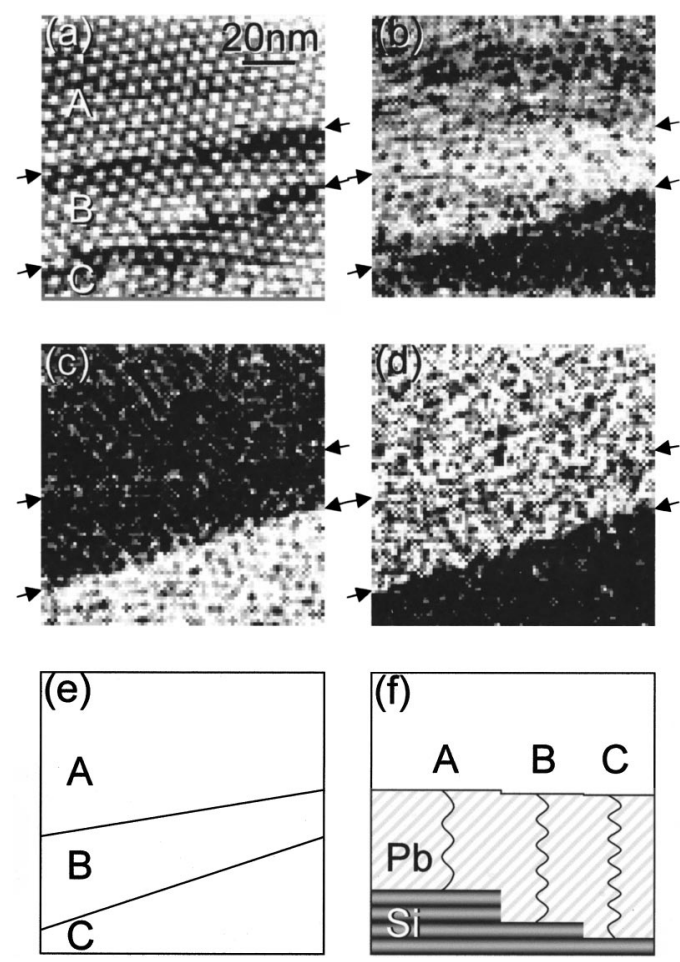

FIG. 2. (a) Constant-current scanning tunneling microscopy image of an approximately 10 monolayer thick $\mathrm{Pb}$ film on $\mathrm{Si}(111) 6 \times 6-\mathrm{Au}$. Two subtle height changes are marked by arrows. (b) - (d) $d I / d V$ images for voltage ranges of (b) -0.07 to -0.19 , (c) +0.03 to +0.26 , and (d) +0.38 to +0.54 V. (e) and (f) show schematically the positions and heights in top view and cross-sectional view, respectively, of the step structure at the buried interface in the area shown in frame (a). The three terraces at the interface are marked by $\mathrm{A}, \mathrm{B}$, and $\mathrm{C}$.

into three regions labeled A, B, and C. Upon close examination, one finds very small step heights $(\sim 0.02-0.05 \mathrm{~nm})$, about a factor of 10 smaller than the regular step heights for $\mathrm{Pb}$ (111) surface observed in Fig. 1. While these tiny surface steps imply a likely existence of underlying steps due to the mismatch in the layer thickness between $\mathrm{Si}(111)$ and the $\mathrm{Pb}(111)$, it is impossible to determine the actual step heights at the interface based on STM images. As we discuss later, the scanning tunneling spectroscopy allows us to accurately determine the underlying step structures.

Figure 3 shows three $(d I / d V) /(I / V)$ spectra measured on the three different surface areas [labeled A, B, and C in Fig. 2(a)] separated by the subtle lines of darker contrast. The spectra exhibit peaks in the density of states. These peaks arise from quantum well states induced by the spatial confinement of the thin $\mathrm{Pb}$ film. ${ }^{9-11}$ The energy separation between neighboring quantum well states, $\Delta$, is decreasing from spectra $\mathrm{A}$ to $\mathrm{C}$. From this we can conclude that the thickness of the $\mathrm{Pb}$ film, i.e., the width of the confining potential, is increasing from spectra A to C. Thus, between the upper part of the STM image in Fig. 2(a) and its lower part at least two steps must be present at the buried $\mathrm{Pb}-\mathrm{Si}$ interface in order to increase the thickness of the $\mathrm{Pb}$ film.

These buried steps can be directly observed using spectroscopic imaging. We extracted from the individual currentvoltage spectra the value of $d I / d V^{14}$ and plotted this as a function of the position on the surface for three different energy intervals [Figs. 2(b)-2(d)]. The three energy intervals were chosen such that a clear contrast is obtained between Downloaded 15 Dec 2006 to 134.94.122.39. Redistribution subject

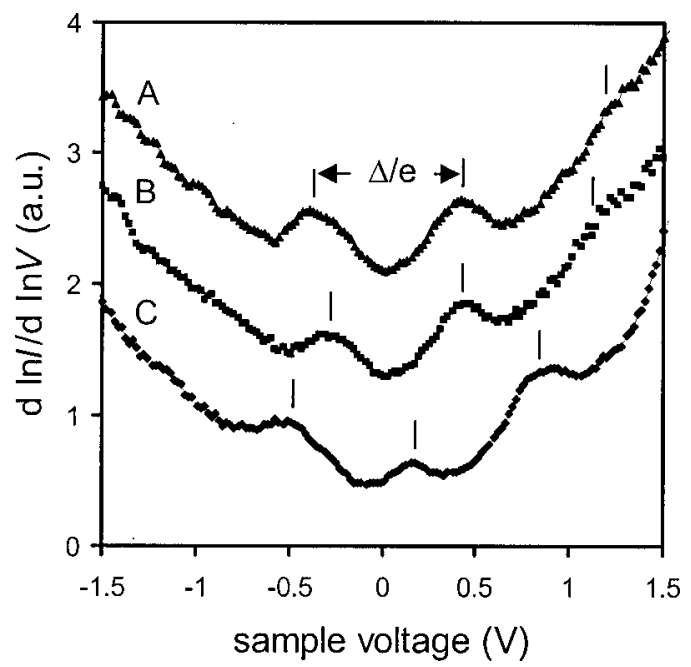

FIG. 3. $(d I / d V) /(I / V)$ spectra derived from current vs voltage spectra of the three areas labeled A, B, and C in Fig. 2(a). The dashes indicate the positions of the quantum well states.

the three different surface regions labeled A-C in Fig. 2(a). This is achieved by choosing three different energy intervals, which each contain one QWS (peak in the spectra of Fig. 3) in one of the three different surface areas, respectively. As a consequence, different contrasts [density of states high (low) for bright (dark) contrast] are obtained for the different surface areas. In these three $d I / d V$ images one can see two boundaries (marked by arrows). The lower one exhibits a pronounced switch of the density of states from lower to higher [Fig. 2(c)] or higher to lower one [Figs. 2(b) and 2(d)]. The other boundary, although hardly observable in Figs. 2(c) and 2(d), leads to a weak, albeit discernable contrast in Fig. 2(b). Since the $d I / d V$ images arise from different positions of the quantum well states, they show changes in the film thickness arising from steps at the $\mathrm{Pb}-\mathrm{Si}$ interface. Thus, one can conclude that two steps are present and their spatial positions are visible in the $d I / d V$ images. The schematic step positions are shown in Fig. 2(e).

In order to obtain a full three-dimensional information of the interface step structure, we need to extract the heights of the steps. We obtain this information from the spectra in Fig. 3. We proceed as follows: First we use the energy difference between neighboring quantum well states to extract the relative thickness changes in the $\mathrm{Pb}$ film. Second the spectra allow us to extract if a given step height is an even or odd number of $\mathrm{Si}(111)$ layers. The energy difference between neighboring quantum well states near the Fermi energy is given by $\Delta=\left(\pi \hbar \nu_{F}\right) /\left(N a_{0}\right),{ }^{9}$ with $N$ being the number of layers of the metal film, $a_{0}$ the thickness of a layer, and $\nu_{F}$ the Fermi velocity of $1.9 \times 10^{6} \mathrm{~m} / \mathrm{s} .{ }^{9}$ The energy differences $\Delta$ between two quantum well states are $0.785 \pm 0.030$, $0.705 \pm 0.030$, and $0.655 \pm 0.030 \mathrm{eV}$ derived from the spectra A to $\mathrm{C}$, respectively. Using these values and the above equation we obtain for the step from the area A to B and B to C a height of $2 \pm 1$ and $1.5 \pm 1$ monolayers, respectively. Next we base our analysis on the absolute peak positions in Fig. 3 . As the number of layers in the $\mathrm{Pb}$ film varies, the states near the Fermi level shift in energy. The energy shift is given by $\delta=\left(2 a_{0} / \lambda_{F}\right) \Delta$. Since the Fermi wavelength $\lambda_{F}$ is close to four times of $a_{0},{ }^{9}$ the energy of a quantum well close to the o AlP license or copyright, see http://apl.aip.org/apl/copyright.jsp 
Fermi energy shifts by about $\Delta / 2$ if the thickness of the metal film changes by one monolayer. As a consequence two metal films with a thickness of $N$ and $N+2$ monolayers exhibit essentially the same peak positions (energy positions of the quantum well states). ${ }^{9,11}$ Only a slight increase in the peak separation is found. Applying this to our spectra, we can conclude that the spectra A and B must arise from metal films whose thicknesses differ by an even number of monolayers, while spectrum $\mathrm{C}$ as compared to spectra A and B differ by an odd number of monolayers in the film thickness. Combining the information about the energy separation and peak position we can extract that the step between areas A and $\mathrm{B}$ is 2 monolayers high, whereas the step between areas $\mathrm{B}$ and $\mathrm{C}$ is 1 monolayer high. Adding the trend that the peak separation is increasing from spectra $\mathrm{C}$ to $\mathrm{A}$, we know that the thickness of the $\mathrm{Pb}$ film increases from $\mathrm{A}$ to $\mathrm{C}$. Thus, the underlying step structure at the buried interface is given by the schematic drawing in Fig. 2f.

Finally, the slight corrugation in the constant-current STM image at the steps' position arises from the lattice mismatch along the $\mathrm{Si}(111)$ and $\mathrm{Pb}(111)$ direction $[$ the $\mathrm{Pb}$ film has a (111) orientation]. The lattice spacings are $0.313 \mathrm{vs}$ $0.286 \mathrm{~nm}$ for $\mathrm{Si}$ and $\mathrm{Pb}$, respectively, yielding a height offset at the surface of 0.027 and $0.054 \mathrm{~nm}$ for a single and double height step at the interface. These values are in good agreement with the height change observed in the constant-current STM image.

In conclusion, we demonstrated that one can determine with atomic layer precision the three-dimensional step structure at buried metal-semiconductor interfaces even for fully covering flat two-dimensional thin film morphologies by exploiting the presence and thickness dependence of quantized electron states in the metal film. For the whole procedure, we do not need to know the exact film thickness, which was close to 10 monolayers in our case. Neither do we need to measure the height of any island. As a consequence the methodology can be applied to any kind of buried metalsemiconductor interface and, as long as quantum well states are present due to a confinement between the buried interface and the vacuum barrier, also to metal-metal heterointerfaces.

This work was supported by NSF Grant Nos. DMR0071893, CHE-8920120, and CTS-9512657, and by the Texas Advanced Research Program.

${ }^{1}$ A. R. Smith, K.-J. Chao, Q. Niu, and C.-K. Shih, Science 273, 226 (1996).

${ }^{2}$ D. A. Luh, T. Miller, J. J. Paggel, M. Y. Chou, and T. C. Chiang, Science 292, 1131 (2001).

${ }^{3}$ Z. Zhang, Q. Niu, and C.-K. Shih, Phys. Rev. Lett. 80, 5381 (1998).

${ }^{4}$ K.-J. Chao, Z. Zhang, Ph. Ebert, and C.-K. Shih, Phys. Rev. B 60, 4988 (1999).

${ }^{5}$ H. Yu, C. S. Jiang, Ph. Ebert, X. D. Wang, J. M. White, Q. Niu, Z. Zhang, and C. K. Shih, Phys. Rev. Lett. 88, 016102 (2002).

${ }^{6}$ I. Matsuda, H. W. Yeom, T. Tanikawa, K. Tono, T. Nagao, S. Hasegawa, and T. Ohta, Phys. Rev. B 63, 125325 (2001).

${ }^{7}$ D. A. Evans, M. Alonso, R. Cimino, and K. Horn, Phys. Rev. Lett. 70, 3483 (1993).

${ }^{8}$ C.-S. Jiang, H.-B. Yu, X.-D. Wang, C.-K. Shih, and Ph. Ebert, Phys. Rev. B 64, 235410 (2001).

${ }^{9}$ I. B. Altfelder, K. A. Matveev, and D. M. Chen, Phys. Rev. Lett. 78, 2815 (1997).

${ }^{10}$ I. B. Altfelder, D. M. Chen, and K. A. Matveev, Phys. Rev. Lett. 80, 4895 (1998).

${ }^{11}$ W. B. Su, S. H. Chang, W. B. Jian, C. S. Chang, L. J. Chen, and T. T. Tsong, Phys. Rev. Lett. 86, 5116 (2001).

${ }^{12}$ G. Hormandinger and J. B. Pendry, Surf. Sci. 295, 34 (1993).

${ }^{13}$ Th. Schmidt and E. Bauer, Phys. Rev. B 62, 15815 (2000).

${ }^{14}$ Plotting the value $(d I / d V) /(I / V)$ yields the same results as both are approximations of the density of states. 